\section{Regards sur l'économie allemande}

Bulletin économique du CIRAC

77 | 2006

Varia

\title{
Préparation $\mathrm{du} 7^{\mathrm{e}} \mathrm{PCRD}$ : les priorités de l'Allemagne
}

\author{
Marie-Hélène Pautrat
}

\section{Q OpenEdition}

Journals

Édition électronique

URL : http://journals.openedition.org/rea/799

DOI : $10.4000 /$ rea.799

ISBN : 978-2-8218-0850-8

ISSN : 1965-0787

Éditeur

CIRAC

Édition imprimée

Date de publication : 1 juillet 2006

Pagination : 25-28

ISSN : 1156-8992

Référence électronique

Marie-Hélène Pautrat, "Préparation du 7e PCRD : les priorités de l'Allemagne », Regards sur l'économie allemande [En ligne], 77 | juillet 2006, document 4, mis en ligne le 01 juillet 2008, consulté le 25 avril 2019. URL : http://journals.openedition.org/rea/799; DOI : 10.4000/rea.799 


\title{
Préparation du $7^{\mathrm{e}}$ PCRD : les priorités de 1'Allemagne
}

\author{
Marie-Hélène Pautrat
}

\begin{abstract}
Engagée dès septembre 2003, la préparation du 7 Programme-Cadre de Recherche et de Développement technologique de l'Union européenne, principal instrument d'une politique de recherche communautaire, entre aujourd'hui dans sa phase finale. Les premiers appels à proposition devraient être publiés début 2007, c'est-à-dire au moment où l'Allemagne prendra la présidence de l'Union européenne. S'il s'inscrit explicitement dans la continuité du précédent, le 7 PCRD sera doté de moyens plus ambitieux pour mieux contribuer aux objectifs de renforcement de la compétitivité (stratégie de Lisbonne) et accompagner les politiques communautaires. L'Allemagne a donc accueilli favorablement les propositions initiales de la Commission européenne, avec quelques nuances néanmoins quant à certains éléments nouveaux du programme. Un rappel des priorités allemandes en matière de recherche européenne s'avère utile au moment où le gouvernement annonce qu'il placera la recherche et l'innovation au centre de sa présidence de l'UE en 2007.
\end{abstract}

Premier bénéficiaire à ce jour des financements de l'UE dans le cadre du $6^{\mathrm{e}}$ PCRD en cours (près de $20 \%$ ), premier pays au regard du nombre de participations financées (4 496) et du nombre de coordinations de projets (36), l'Allemagne a su bénéficier pleinement du dispositif européen de financement de la recherche. Les propositions en vue du lancement du $7^{e}$ PCRD vont dans le sens d'un renforcement des actions du précédent programme-cadre, avec toutefois une durée portée à 7 ans (au lieu de 5) et un effort financier accru. Surtout, ce $7^{\mathrm{e}}$ PCRD propose des actions nouvelles : la mise en place d'un Conseil européen de la recherche, des initiatives technologiques conjointes et un soutien accru à la création d'infrastructures de recherche. Considéré comme un moyen de renforcer la compétitivité européenne, cet instrument est en adéquation avec la conception actuelle de la recherche et de l'innovation défendue par l'Allemagne. Les premières orientations de la Commission (COM(2004) $353 \mathrm{du}$ 16-06-2004) ont globalement été favorablement accueillies par l'Allemagne qui s'est prononcée sur la structure et le contenu du $7^{\mathrm{e}}$ PCRD dans son document de référence Advancing the European Research Area!. Cette position, ellemême résultat de consultations menées avec les acteurs de la recherche et des milieux économiques, a été précisée ultérieurement dans différents documents portant sur des points jugés prioritaires, à mesure que progressait le processus de consultation et de concertation européen. Mais pour l'Allemagne, ce nouveau programme-cadre devra répondre à trois objectifs : poursuivre la structuration de l'Espace européen de Recherche, prendre en compte les objectifs de Lisbonne, apporter un soutien aux politiques communautaires.

La très sensible augmentation de la dotation financière constitue la caractéristique majeure de ce nouveau PCRD. Alors que $6^{e}$ PCRD est doté d'un budget de 17,5 milliards $€$ (y compris le programme EURATOM de recherche et formation dans le domaine nucléaire) pour la période 2002-2006, la Commission proposait en avril 2005 une forte augmentation à 72726 millions € pour le $7^{\mathrm{e}}$ PCRD (2007-2013). En mars dernier, un compromis institutionnel avec le Conseil et le Parlement européen a finalement ramené cette enveloppe à
Advancing the

European Research Area!

Un effort financier en nette augmentation 
Les priorités thématiques au cœur du PCRD

50 milliards $€$ (4 milliards supplémentaires pour EURATOM), à répartir entre 4 grands types d'activités spécifiques : 32 milliards $€$ affectés au programme COOPERATION (finançant la recherche collaborative sur des thématiques proposées, les plates-formes technologiques et initiatives technologiques conjointes, ainsi que la coordination des programmes de recherche nationaux), 7,5 milliards $€$ au programme IDEES (soutien à la recherche fondamentale par la création du Conseil européen de la Recherche), 5 milliards $€$ au volet PERSONNEL (développer les ressources humaines en R\&D en Europe) et 4,2 milliards $€$ au programme CAPACITES (développement des infrastructures de recherche). Pour sa part, le gouvernement allemand s'est prononcé pour un accroissement du budget du PCRD, salué par les acteurs allemands de la recherche. L'Allemagne adhère en effet pleinement à l'objectif du Conseil européen de Barcelone (2002) d'augmenter les dépenses pour la recherche et l'innovation à $3 \%$ du PIB européen en 2010. Mais cette hausse du budget alloué au PCRD doit respecter la limite d'une contribution financière des Etats membres au budget de I'UE plafonnée à $1 \%$ du PIB. Par ailleurs, les deux tiers de l'effort financier de R\&D devant être réalisés par les entreprises selon les objectifs de Barcelone, l'Allemagne considère que le $7^{e}$ PCRD devra constituer pour les PME un levier pour tenter d'accroître leurs dépenses de R\&D.

Au cœur du PCRD, le volet COOPERATION vise à renforcer la recherche collaborative à l'échelle européenne autour de priorités thématiques, afin de créer des pôles d'excellence européens par la coopération entre laboratoires. Ces priorités, déjà recentrées dans le $6^{\mathrm{e}} \mathrm{PCRD}$, doivent, selon la position allemande, être renforcées, également au plan financier. Le gouvernement avait précisé en février 2005 neuf grands champs thématiques identifiés, selon lui, comme des domaines porteurs susceptibles de renforcer la compétitivité européenne et devant faire l'objet d'un effort particulier. Chaque projet soutenu dans le cadre des priorités thématiques finalement retenues au terme du processus de concertation à l'échelon européen devra dès lors venir en appui des politiques communes. Par ailleurs, l'Allemagne insiste sur la nécessité de renforcer la capacité de réaction et d'anticipation face à l'émergence de domaines de recherche innovants, en ménageant une marge de financement pour des projets originaux en marge des thématiques proposées.

\section{Priorités thématiques de la RFA : domaines de recherche et activités}

\begin{tabular}{|c|c|c|}
\hline $\begin{array}{l}\text { Sciences du vivant, biotechnologies : } \\
\text {-connaissances fondamentales, outils, } \\
\text { technologies ; } \\
\text {-économie basée sur la biotechnologie ; } \\
\text { - applications dans le domaine de la santé ; } \\
\text {-technologies biomédicales ; } \\
\text {-sciences du vivant et société ; } \\
\text {-biophotonique. }\end{array}$ & $\begin{array}{l}\text { Nanotechnologies, matériaux et } \\
\text { technologies de production : } \\
\text {-nanotechnologies ; } \\
\text {-intégration de systèmes ; } \\
\text {-recherche en productique; } \\
\text {-nouveaux matériaux; } \\
\text {-technologies optiques. }\end{array}$ & $\begin{array}{l}\text { Technologies de l'information et des } \\
\text { communications : } \\
\text {-nanoélectronique ; } \\
\text {-technologies de la communication; } \\
\text { - eScience. }\end{array}$ \\
\hline $\begin{array}{l}\text { Recherche spatiale : } \\
\text {-station spatiale internationale ; } \\
\text {-applications ; observation de la terre ; } \\
\text {-applications : navigation; } \\
\text {-science de l'espace et robotique. }\end{array}$ & $\begin{array}{l}\text { Aéronautique : } \\
\text {-avions plus écologiques, conviviaux pour les } \\
\text { passagers et rentables ; } \\
\text {-systèmes de propulsion peu générateurs } \\
\text { d'émissions polluantes ; } \\
\text {-hélicoptères plus silencieux, plus performants et } \\
\text { résistants à toutes les conditions climatiques ; } \\
\text {-système de transport aérien sûr et plus efficient. }\end{array}$ & $\begin{array}{l}\text { Energie : } \\
\text {-technologies améliorant le rendement énergé- } \\
\text { tique; } \\
\text {-sources d'énergie renouvelables ; } \\
\text {-technologies modernes pour les centrales ther- } \\
\text { miques au charbon et au gaz ; } \\
\text {-piles à combustible ; } \\
\text {-distribution et stockage d'électricité, hydrogène. }\end{array}$ \\
\hline $\begin{array}{l}\text { Transports et mobilité : } \\
\text {-systèmes intégrés paneuropéens de trans- } \\
\text { port ; } \\
\text {-améliorer la division du travail, mieux utiliser } \\
\text { les capacités disponibles dans le transport de } \\
\text { marchandises } \\
\text {-transports durables ; } \\
\text {-recherche dans le domaine de la sécurité ; } \\
\text {-techniques marines. }\end{array}$ & $\begin{array}{l}\text { Développement durable ; Système Terre : } \\
\text {-changement climatique ; } \\
\text {-cycles biogéochimiques : perspectives europé- } \\
\text { ennes et globales; } \\
\text {-protection des ressources naturelles et gestion } \\
\text { socialement et économiquement durable ; } \\
\text {-recherche océanographique, ressources marines, } \\
\text { zones côtières et gestion socialement et écono- } \\
\text { miquement durable ; } \\
\text {-interactions environnement / santé. }\end{array}$ & $\begin{array}{l}\text { Sciences humaines et sociales : } \\
\text {-processus et enjeux d'intégration sociaux; } \\
\text {-globalisation économique et culturelle : perspec- } \\
\text { tives d'innovation; } \\
\text {-relations entre milieux scientifiques, politique } \\
\text { (de recherche) et société ; } \\
\text {-recherche sur la paix et les conflits pour une po- } \\
\text { litique européenne de sécurité. }\end{array}$ \\
\hline
\end{tabular}

Source : BMBW, Thematische Vorstellungen der Bundesregierung für das Europäische Sicherheitsforschungsprogramm im 7. EU-Forschungsrahmenprogramm. Arbeitspapier 
Dans sa communication du 16-06-2004, la Commission proposait des actions spécifiques dans deux nouveaux domaines : l'espace et la sécurité, considérés comme deux enjeux majeurs pour l'Europe. Dans ce contexte, l'Allemagne adhère à une stratégie européenne ayant pour but de soutenir la mise en œuvre de la politique spatiale européenne, en coopération avec l'Agence spatiale européenne. A condition toutefois que le financement dans le cadre du PCRD concerne expressément les travaux de recherche, et non les programmes d'application et de développement. Même réserve du côté du rattachement d'un programme de recherche européen sur la sécurité au $7^{\mathrm{e}}$ PCRD. Celui-ci devrait se limiter strictement à la recherche civile. Par ailleurs, l'Allemagne souhaite que les Etats membres soient impliqués au sein d'un comité de programme.

Les instruments de la recherche proposés par le $6^{\mathrm{e}}$ PCRD dans le cadre des priorités thématiques ont fait l'objet de critiques récurrentes, notamment en raison de leur complexité et de leur manque de lisibilité, avec un effet dissuasif pour les équipes de taille modeste ou les PME. Partageant les recommandations du rapport Marimon, l'Allemagne attache également une importance particulière à ce que le porteur d'un projet puisse décider du choix des instruments, dont le nombre doit rester limité. Dès lors, c'est avec une certaine réserve qu'a été accueillie l'idée de faire évoluer certaines plates-formes technologiques existantes du $6^{\mathrm{e}}$ PCRD vers des initiatives technologiques conjointes (JTI), grands projets à l'échelle européenne pour des domaines où d'importantes avancées technologiques sont envisageables à moyen et long termes, sous réserve d'un fort degré de coordination des efforts à l'échelon européen (hydrogène et piles à combustible ; médecine innovante ; systèmes embarqués ; aéronautique; nanoélectronique; surveillance globale de l'environnement: six domaines identifiés par la Commission). Redoutant des difficultés de management, l'Allemagne recommande donc une phase d'expérimentation avec quelques projets seulement et une réflexion sur l'articulation entre les JTI (fonctionnant selon les principes des programmes-cadre, à savoir l'excellence et la compétition pour les financements) et les projets EUREKA (portés par les industriels selon une approche de marché).

Autre nouveauté notable du $7^{e} \mathrm{PCRD}$, le volet IDEES prévoit la mise en place de l'ERC (Conseil européen de la Recherche), qui répond à une demande de la communauté scientifique. Alors que les précédents programmes-cadres étaient centrés sur la recherche finalisée, l'ERC aura pour mission de stimuler la recherche dite 'exploratoire' par une mise en concurrence des meilleures équipes. Objectif : aider à promouvoir un niveau de reconnaissance internationale en dépassant le morcellement de la recherche fondamentale européenne, tributaire des financements nationaux. Cette idée fait depuis longtemps l'objet d'un large consensus, et c'est donc moins le principe que les modalités d'organisation et de fonctionnement qui sont l'enjeu des discussions. Pour l'Allemagne, le financement de travaux de recherche fondamentale devra être fondé sur l'unique critère de l'excellence, selon les normes admises par la communauté scientifique (Peer Review). La position allemande insiste également sur l'autonomie du futur ERC par rapport à la Commission et aux gouvernements nationaux : les décisions doivent relever exclusivement de critères scientifiques, et il importe d'éviter d'éventuelles dérives (principe de juste retour). L'ERC devra donc définir lui-même sa structure et ses procédures de soutien, souhaitées suffisamment flexibles et réactives par l'Allemagne. Enfin, le financement ne devra pas s'effectuer au détriment des priorités thématiques. D'où l'importance, pour l'Allemagne, d'une mise en oeuvre progressive, d'abord limitée à quelques domaines de recherche.

En dépit des efforts de simplification, la question de la lourdeur bureaucratique des procédures de soumission, de négociation et de gestion des projets, mais également du coût engendré, reste en effet un point majeur des discussions, puisqu'il en va de l'attractivité et de l'acceptation du programme-cadre par les chercheurs, et surtout par les PME. Avec l'initiative SURF (Simpification of
L'espace et la sécurité : nouveaux domaines de recherche

Pour des instruments de la recherche mieux manipulables

L'ERC et le soutien à la recherche exploratoire

Réduire la charge administrative des projets 
European Research Funding), élaborée en concertation avec les grands centres de recherche, l'Allemagne rappelait que l'augmentation du budget alloué au PCRD et l'introduction de nouvelles mesures doivent nécessairement s'accompagner d'une simplification des processus de décision, avançant plusieurs propositions concrètes d'amélioration des règles de participation et d'optimisation de la gestion du programme. Car l'enjeu est double : donner de réelles chances afin d'éviter la mise à l'écart d'excellents projets, comme c'est le cas dans le $6^{\mathrm{e}} \mathrm{PCRD}$, et encourager la participation des PME.

Renforcer la participation allemande aux programmes européens passe aussi par une meilleure participation des entreprises, et notamment des PME à haut potentiel de croissance mais sans activité de recherche intensive. Or beaucoup de PME se sont détournées du $6^{\mathrm{e}}$ PCRD qui a favorisé les projets lourds et structurants, au profit des grands groupes industriels. L'implication des PME dans les programmes européens est en effet étroitement liée aux conditions de participation : thématiques suffisamment larges, simplicité des procédures, possibilité de réaliser des projets de recherche à dimension réduite et réelles perspectives de réussite des projets soumis. Pour cela, l'Allemagne a insisté sur la nécessité de bien préciser les dispositions pour la participation des PME dans le cadre des priorités thématiques, en proposant des outils adaptés aux besoins des entreprises de petite taille (comme les projets spécifiques ciblés STREPS, à dimension plus réduite), en renforçant les instruments spécifiques existants (comme les projets KRAFT, bien utilisés par les PME allemandes) ou en améliorant les synergies avec le programme EUREKA.

MEME SI SA StRuCtURE N'EST PAS DEFINITIVEMENT ADOPTEE, ce $7^{e}$ PCRD se voit accorder un rôle majeur pour tenter de réactiver les objectifs de Lisbonne. Dans la continuité du précédent (structuration de l'Espace européen de la Recherche sur la base d'un double principe de coopération scientifique et de compétition pour les financements), il comporte tout de même des accents nouveaux : renforcer l'attractivité scientifique de l'Europe et accompagner les mutations économiques pour stimuler la compétitivité européenne. Cette inflexion, qui doit se traduire par une compétition selon le seul critère de l'excellence, par un rééquilibrage entre recherche appliquée et recherche fondamentale, et par une plus forte implication de la recherche industrielle, fait écho à la stratégie pour la recherche et l'innovation poursuivie en Allemagne par le gouvernement Merkel : son programme de relance de la croissance consacre 6 milliards $€$ d'ici 2009 à la R\&D. Objectif : soutenir la recherche de pointe et accélérer le passage des résultats de R\&D vers des débouchés commerciaux et, in fine, des emplois. Les entreprises, dont l'effort de R\&D avait fléchi en 2003-2004, et notamment les PME, considérées comme un moteur de l'emploi, seront au centre de cette stratégie. Le lancement officiel du $7^{\mathrm{e}}$ PCRD en janvier prochain devrait donner l'occasion à l'Allemagne de réaffirmer ses choix politiques.

\section{Indications bibliographiques :}

BUNDESMINISTERIUM FüR BILDUNG UND FORSCHUNG (REF. 113), Arbeitspapier zur Konkretisierung der Vorstellungen zu Inhalten der "Thematischen Prioritäten" im 7. EU-Forschungsrahmenprogramm, Bonn, février 2005

BUNDESMINISTERIUM FüR BILDUNG UND FORSCHUNG (REF. 113), Thematische Vorstellungen der Bundesregierung für das Europäische Sicherheitsforschungsprogramm im 7. EUForschungsrahmenprogramm. Arbeitspapier, Bonn, 17.02.2005

BUNDESMINISTERIUM FüR BILDUNG UND FORSCHUNG, Den europäischen Forschungsraum voranbringen! Kernforderungen der Bundesregierung an das 7. EU-Forschungsrahmenprgoramm, Bonn, 26.11.2004

BUNDESMINISTERIUM FüR BILdUNG UNd FoRSCHUng (Ref. 113), Vereinfachung der Förderverfahren im 7. EU-Forschungsrahmenprogramm - SURF, mai 2005

CoMmission EUROPÉENNE, La science et la technologie, clés de l'avenir de l'Europe. Orientations pour la politique de soutien à la recherche de l'Europe, COM (2004) $353 \mathrm{du}$ 16-06-2004

www.rp6.de/inhalte/rp7/ : documents officiels de l'Allemagne 